\title{
Parâmetros Genéticos para a Produção de Leite de Controles Individuais de Vacas da Raça Gir Estimados com Modelos de Repetibilidade e Regressão Aleatória1 ${ }^{1}$
}

\author{
Claudio Napolis Costa ${ }^{2}$, Claudio Manoel Rodrigues de Melo $^{3}$, Carlos Henrique Crivelari Machado4, \\ Ary Ferreira de Freitas ${ }^{2}$, Irineu Umberto Packer ${ }^{5}$, Jaime de Araújo Cobuci ${ }^{6}$
}

\begin{abstract}
RESUMO - Registros individuais perfazendo 8.183 controles de produção de leite (PLC) de 1.273 primeiras lactações de vacas da raça Gir de rebanhos supervisionados pela ABCZ no período 1994/2000 foram utilizados para se estimar componentes de variância e parâmetros genéticos para PLC usando-se REML. Foram comparados um modelo de repetibilidade e modelos de regressão aleatória ajustados com as funções logarítmica (Ali \& Schaeffer, 1987), exponencial (Wilmink, 1987) e polinômios ortogonais de Legendre (LP) de ordem 3 a 5, sob as pressuposições de homogeneidade e heterogeneidade de variância residual, definidas respectivamente por uma $(\mathrm{ME}=1)$ ou quatro classes de medidas de erro temporário $(\mathrm{ME}=4)$ ao longo do período de lactação. Também ajustou-se a produção de leite acumulada na lactação (P305) utilizando-se um modelo animal cuja estimativa de heritabilidade foi 0,22. As estimativas de heritabilidade e repetibilidade para a PLC foram 0,27 e 0,76 , respectivamente. As estimativas de heritabilidade com a FAS e a FW alcançaram respectivamente 0,59 e 0,74 ao início da lactação e decresceram para valores próximos de 0,20 ao final do período. Exceto para o LP de quinta ordem com $\mathrm{ME}=1$, as estimativas de heritabilidade diminuíram de 0,70 no início para 0,30 no final da lactação. Menores estimativas de VR foram obtidas para a FAS do que para a FW sob ambas as pressuposições de homogeneidade e heterogeneidade de variância. Em todos os estádios da lactação, as estimativas de VR diminuíram com o aumento da ordem do LP e dependeram da pressuposição sobre o ME. As estimativas das variâncias genética e de ambiente permanente não apresentaram nenhuma tendência com o aumento da ordem do LP e não se observaram diferenças significativas para tais estimativas sob a pressuposição de heterogeneidade de VR ao longo da lactação. Maiores valores de correlação genética entre as PLC foram obtidos com a FW, que também apresentou maior número de estimativas negativas entre as PLC do início e fim da lactação do que a FAS. Exceto para a FAS, observou-se redução das estimativas de correlação genética próximas à unidade entre as PLC adjacentes para valores negativos entre as PLC no início e no fim da lactação. Entre os polinômios de Legendre, o de quinta ordem apresentou um melhor o ajuste das PLC. Os resultados indicam o potencial de uso de regressão aleatória, com os modelos LP5 e a FAS apresentando-se como os mais adequados para a modelagem das variâncias genética e de efeito permanente das PLC da raça Gir.
\end{abstract}

Palavras-chave: avaliação genética, componentes de variância, heritabilidade, seleção, zebu

\section{Estimation of Genetic Parameters for Test Day Milk Records of First Lactation Gyr Cows Using Repeatability and Random Regression Animal Models}

\begin{abstract}
Data comprising 8,183 test day records of 1,273 first lactations of Gyr cows from herds supervised by ABCZ were used to estimate variance components and genetic parameters for milk yield using repeatability and random regression animal models by REML. Genetic modelling of logarithmic (FAS), exponential (FW) curves was compared to orthogonal Legendre polynomials (LP) of order 3 to 5 . Residual variance was assumed to be constant in all $(\mathrm{ME}=1)$ or some periods of lactation (ME=4). Lactation milk yield in 305 $\mathrm{d}$ was also adjusted by an animal model. Genetic variance, heritability and repeatability for test day milk yields estimated by a repeatability animal model were $1.74 \mathrm{~kg} 2,0.27$, and 0.76 , respectively. Genetic variance and heritability estimates for lactation milk yield were respectively $121,094.6$ and 0.22 . Heritability estimates from FAS and FW, respectively, decreased from 0,59 and 0.74 at the beginning of lactation to 0.20 at the end of the period. Except for a fifth-order LP with ME=1, heritability estimates decreased from around 0,70 at early lactation to 0,30 at the end of lactation. Residual variance estimates were slightly smaller for logarithimic than for exponential curves both for homogeneous and heterogeneous variance assumptions. Estimates of residual variance in all stages of lactation decreased as the order of LP increased and depended on the assumption about ME. Estimates of genetic and permanent environment variances did not show any trend due to the increase in the order of LP. Genetic correlation estimates between TD were largest for LP and larger for FW than for FAS. Except for FAS, a similar pattern in genetic correlation estimates was observed for all curves decreasing from values close to unity between adjacent TD at early lactation to negative values for TD in the beginning and the end of lactation. The Legendre polynomial of order five and the FAS under ME4 best fitted the data. There is a potential for using random regression to model animal genetic and permanent environmental effects using test day information of Gyr cows.
\end{abstract}

Key Words: genetic evaluation, heritability, selection, variance components, zebu

\footnotetext{
${ }^{1}$ Pesquisa parcialmente financiada pela Fapemig, Projeto CAG 1138/98.

2 Embrapa Gado de Leite, 36038-330 Juiz de Fora, MG (cnc8@cnpgl.embrapa.br)

3 UFSC, Departamento de Aqüicultura, 88040-900 Florianópolis, SC.

${ }^{4}$ Associação Brasileira de Criadores de Zebu, 38022-330 Uberaba, MG

${ }^{5}$ Esalq/USP, Departamento de Produção Animal, 13418-900 Piracicaba, SP.

${ }^{6}$ UFRGS, Departamento de Zootecnia, 90001-970 Porto Alegre, RS.
} 


\section{Introdução}

A avaliação genética de bovinos para a produção de leite têm sido baseada na produção acumulada na lactação com um período padrão de 305 dias, cujo total é estimado a partir das produções medidas nos dias dos controles leiteiros, geralmente realizados em intervalos de 30 dias. Avanços no desenvolvimento de algoritmos e recursos computacionais têm possibilitado o ajuste de modelos para a produção do dia do controle (PLC) como alternativa aos modelos de ajuste da produção acumulada da lactação em até 305 dias (P305).

Algumas vantagens têm sido atribuídas aos modelos para ajuste da PLC, destacando-se a definição mais precisa dos grupos contemporâneos e dos efeitos ambientais à eles associados, o melhor ajuste para lactações de diferentes durações e portanto um descrição mais especifica dos efeitos de estágio de lactação dos animais em produção (Ptak \& Schaeffer, 1993; Swalve, 1995). Outros aspectos associados ao uso da PLC são a possibilidade de avaliação de animais com lactações em curso ou parciais, o que viabiliza a realização de avaliações mais freqüentes, e assim, a redução do intervalo de gerações (Hill et al., 1995); a flexibilidade quanto ao sistema de controle leiteiro e, ainda, a perspectiva de maior precisão nas estimativas de valor genético, ao se ter disponível maior número de observações por animal (Swalve, 2000; Jensen, 2001).

Diversos modelos têm sido avaliados para ajuste das PLC e não existe ainda um consenso sobre qual o modelo mais apropriado para as avaliações genéticas. As funções de covariância e os modelos de regressão aleatória permitem modelar a estrutura de covariância entre as PLC, minimizando os problemas associados com um estrutura simplificada dos modelos de repetibilidade ou a superparametrização nos modelos multivariados (Jamrozik \& Schaeffer,1997; Meyer, 1998a; Pool et al., 2000; Swalve, 2000; Jensen, 2001). A estrutura de covariância das medidas repetidas das PLC podem ser caracterizadas por um função de covariância, que é equivalente às regressões aleatórias se a mesma função é usada (Meyer \& Hill, 1997; Meyer, 1998b). Assim, as regressões aleatórias para ajuste da PLC, baseadas em funções paramétricas, caracterizam-se como alternativas interessantes ao permitir a modelagem da curva de lactação, simultaneamente à caracterização de covariâncias entre os registros sucessivos (Olori et al., 1999; Swalve, 2000; Brotherstone et al., 2000) e ainda permitir a realização de avaliações genéticas para a persistência da lactação (Jakobsen et al., 2002).

Os estudos com modelos de regressão aleatória têm recebido uma atenção considerável nos países com programas de seleção bem estruturados. No Brasil, os programas de seleção das raças zebuínas baseados em teste de progênie com a inseminação artificial (Costa et al., 2000; Verneque et al., 2000a) têm sido bem sucedidos, mas são caracterizados por pequena população de animais e por conseguinte reduzido número de lactações para as decisões nos procedimentos de avaliação de mérito genético dos animais. Neste contexto, a perspectiva de se utilizarem nas avaliações genéticas os modelos de ajuste da PLC, que são em maior número que as próprias lactações acumuladas, pode ser uma alternativa interessante, pelo seu potencial de maior precisão nas estimativas de valor genético e correspondente progresso genético pela seleção.

O objetivo deste estudo foi estimar componentes de variância e parâmetros genéticos e comparar o ajuste dos registros da produção de leite do dia do controle de primeiras lactações de vacas Gir com um modelo de repetibilidade e modelos alternativos de regressão aleatória usando-se as funções paramétricas logarítmica e exponencial e os polinômios ortogonais de Legendre.

\section{Material e Métodos}

Foram utilizados 15.328 registros da produção de leite no dia do controle leiteiro (PLC) de 2.095 primeiras lactações de vacas Gir no período de 1990/2000 de rebanhos supervisionados pelo Serviço de Controle Leiteiro da Associação Brasileira de Criadores de Zebu (ABCZ). Foram editados os controles de produção de leite registrados a partir do quinto dia de lactação, com intervalos sucessivos inferiores a 45 dias, de vacas com idade entre 24 e 60 meses e ano de parto entre 1994 e 2000. Em adição, condicionou-se a disponibilidade de grupo contemporâneo caracterizado por rebanho-ano-mês de controle (RAM) com no mínimo quatro vacas. Após essas edições, o arquivo final para as análises compreenderam 8.183 registros de produção de leite de 1.273 vacas, filhas de 239 touros, em 69 rebanhos com média e desvio padrão da produção de leite dos controles iguais a 8.5 e $3.5 \mathrm{~kg}$, 
respectivamente. $\mathrm{O}$ arquivo de pedigree incluiu as informações de genealogia de vacas e touros.

No contexto dos objetivos deste estudo, foram analisados os seguintes modelos:

1. Produção de leite em 305 dias (P305):

O modelo utilizado foi:

$$
y_{i j k}=R A_{i}+E_{j}+\sum_{n=1}^{2} b_{n} x_{i j k}+a_{i j k}+e_{i j k}
$$

em que: $y_{i j k}$ é a produção de leite em até 305 dias de lactação (P305); RA é o efeito do rebanho-ano de parto $i$; $\mathrm{E}_{\mathrm{j}}$ é a época do parto $j, b_{1} \mathrm{e} b_{2}$ são os coeficientes de regressão linear e quadrático da P305 em função da idade da vaca ao parto; $x_{i j k}$ é a idade da vaca ao parto, em dias; $a_{i j k}$ é o efeito genético aditivo aleatório do animal $k$ pertencente ao rebanho-ano de parto $i$ e a época de parto $j$; e $e_{i j k}$ é o erro aleatório associado a cada observação. Os componentes de variância foram estimados com o programa MTDFREML (Boldman et al., 1995) e o critério de convergência foi definido pelo valor do quadrado das diferenças entre estimativas consecutivas menor que $b_{1}$.

2. Modelo de repetibilidade (MR) para a PLC:

O modelo utilizado foi:

$$
y_{i j k l}=R A M_{i}+E_{j}+\sum_{n=1}^{2} b_{n} x_{i j k}+\sum_{n=1}^{4} q_{n} z_{n k l}+a_{i j k}+p e_{i j k}+e_{i j k l}
$$

em que: $y_{i j k}$ é a l-ésima observação de PLC registrada no dia de lactação $t$ da vaca k; RAM é o efeito do rebanho-ano-mês do controle $i$; $\mathrm{E}_{\mathrm{j}}$ é a época do parto $\mathrm{j} ; \sum$ representa o somatório, $b_{1}$ e $b_{2}$ são os coeficientes de regressão linear e quadrático da PLC em função da idade da vaca ao parto; $x_{i j k}$ é a idade da vaca ao parto, em dias; é o efeito genético aditivo aleatório do animal $k$ pertencente ao rebanho-ano-mês do controle $i$ e a época de parto $j$; é o efeito permanente da vaca pertencente ao $\mathrm{RAM}_{\mathrm{i}}$ e a $\mathrm{E}_{\mathrm{j}}$; e $p e_{i j k}$ é o erro aleatório associado a cada observação de PLC; $q_{1}$ a $q_{4}$ são os coeficientes de regressão da PLC em função das covariáveis $z_{n k l}$ que descrevem a curva da lactação, em que: $\quad z_{1 k l}=c ; \quad z_{2 k l}=c^{2} ; \quad z_{3 k l}=\ln (1 / c)$; $z_{4 k l}=(\ln (1 / c))^{2}$ e $\mathrm{c}=\mathrm{t} / 305$ com $\mathrm{t}=$ dias em lactação. Os componentes de variância foram estimados com o programa MTDFREML (Boldman et al., 1995) e o critério de convergência foi definido pelo valor do quadrado das diferenças entre estimativas consecutivas menor que $10^{-9}$.

3. Modelos de regressão aleatória para ajuste da PLC com as funções polinomiais:

O modelo utilizado foi:

$$
\begin{aligned}
& y_{\mathrm{ijk}}=\mathrm{RAM}+\mathrm{E}+\sum \mathrm{b}_{\mathrm{n}} \mathrm{x}_{\mathrm{j} k}+\sum \beta_{m} \phi \mathrm{m}(\mathrm{t})+\sum \alpha_{m} \phi \mathrm{m}(\mathrm{t})+\sum \gamma_{m} \phi \mathrm{m}(\mathrm{t})+\mathrm{e}_{\mathrm{j} k \mathrm{l}} \\
& \mathrm{n}=1 \quad \mathrm{~m}=0 \quad \mathrm{~m}=0 \quad \mathrm{~m}=0
\end{aligned}
$$

em que $y_{\mathrm{ijkl}}$ é a l-ésima observação registrada no dia de lactaçã̃o t do animal k no RAM i, E é a estação j e é a idade da vaca $\mathrm{k}$ ao parto, em meses, como covariável com componentes $n(n=1,2)$ linear e quadrático, $\sum$ representa o somatório e $\beta_{m}, \alpha_{m}$ e $\gamma_{m}$ são coeficientes de regressão aleatória para os efeitos fixos e efeitos aleatórios genético aditivo e de ambiente permanente respectivamente, associados aos coeficientes específicos de cada função polinomial $\left(\phi_{\mathrm{m}}, \mathrm{m}=0, \mathrm{p}-1\right.$ para $\mathrm{p}$ indicando a ordem do polinômio) e $\mathrm{e}_{\mathrm{ijkl}}$ é o erro aleatório ou medida de erro temporário associado a cada PLC.

Foram realizadas quatro análises para a comparação de dois polinômios caracterizados pela seguintes funções:

a) Função logarítmica de Ali \& Schaeffer (1987) caracterizada por cinco parâmetros e representada por FAS é definida por:

$a_{0}+a_{1} c+a_{2} c^{2}+a_{3}(\ln 1 / c)+a_{4}(\ln 1 / c)^{2}$, em que $c=t / 305$ e $t$ é dias em lactação.

b) A função exponencial de Wilmink (1987) caracterizada por quatro parâmetros e representada por FW é definida por:

$a_{0}+a_{1} t+a_{2} \exp \left(-a_{3} t\right)$, em que $t$ é dias em lactação.

Na curva de Wilmink, $a_{3}$ o parâmetro é geralmente considerado como uma constante, para a qual foi assumido o valor para $a_{3}=-0,05$ nesse estudo reduzindo o número de parâmetros a serem estimados de quatro para três, simplificando o ajuste desta curva.

Duas formas de ajuste do efeito residual foram avaliadas para cada função polinomial. Em uma forma assumiu-se variância residual (VR) constante ao longo da lactação (ME1) e, na outra forma assumiu-se que a VR era constante dentro, mas heterogênea entre classes de período de lactação, definidas nos intervalos: 549, 50-119, 120-169 e 170-305 dias de lactação (ME4).

$\mathrm{O}$ modelo anterior pode ser escrito na forma matricial por: 


$$
\mathrm{y}=\mathrm{Xb}+\mathrm{Za}+\mathrm{Z}_{\mathrm{D}} \gamma+\mathrm{e}
$$

em que y é o vetor das observações; $b$, o vetor dos efeitos fixos; a, o vetor dos coeficientes de regressão aleatória do efeito genético aditivo de animal; $\gamma, o$ vetor dos coeficientes de regressão aleatória do efeito permanente de ambiente; e, o vetor de efeito aleatório residual; e $\mathrm{X}, \mathrm{Z}$ e $\mathrm{Z}_{\mathrm{d}}$ são as matrizes de incidência correspondentes às observações, para efeitos fixos, efeitos aleatórios de animal e permanente de ambiente, respectivamente, para os quais assume-se:

$$
\left[\begin{array}{c}
\boldsymbol{\alpha} \\
\boldsymbol{\gamma} \\
\mathbf{e}
\end{array}\right] \sim N(\mathbf{0}, \mathbf{V}), \mathrm{V}=\left[\begin{array}{ccc}
\Lambda_{\mathrm{A}} \otimes \mathrm{A} & 0 & 0 \\
0 & \Lambda_{\mathrm{p}} \otimes \mathrm{I} & 0 \\
0 & 0 & \mathrm{R}
\end{array}\right],
$$

em que $\Lambda_{\mathrm{A}}$ e $\Lambda_{\mathrm{P}}$ são, respectivamente, as matrizes de (co)variância genética aditiva e de efeito permanente de ambiente para os coeficientes de regressão aleatória, A é a matriz dos coeficientes de parentesco entre os animais, I uma matriz identidade, $\otimes$ é o operador de produto direto entre matrizes, e $\mathrm{R}=\operatorname{diag}\left\{\sigma_{e s}^{2}\right\}$, em que $s$ representa as classes de dias em lactação, para ajuste da variância heterogênea entre grupos ao longo da lactação. Assim, no ajuste da curva AS $s=1, \ldots, 4$ para as classes: 5-49, 50-119, 120-169 e 170-305 dias de lactação ou ainda $s=1$ quando assume-se variância residual homogênea ou constante ao longo da lactação. Os modelos ajustados usando a função AS foram identificados por FAS seguido do número de medidas de erro ajustadas (4 ou 1). Assim FAS4 identifica um MRA usando a FAS com ajuste de 4 classes ou medidas de erro.

4. Modelo de regressão aleatória para ajuste da PLC com os polinômios de Legendre (LP):

O modelo utilizado foi:

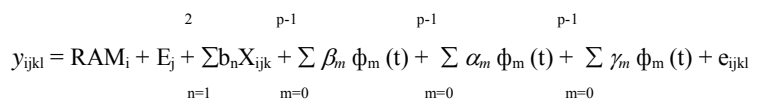

em que yijkl é a l-ésima observação registrada no dia de lactação t do animal k no RAM i, E é a estação j e é a idade da vaca $\mathrm{k}$ ao parto, em meses, como covariável com componentes $\mathrm{n}(\mathrm{n}=1,2)$ linear e quadrático, $\sum$ representa o somatório e $\beta_{m}, \alpha_{m}$ e $\gamma_{m}$ são coeficientes de regressão aleatória para os efei- tos fixos e efeitos aleatórios genético aditivo e de ambiente permanente respectivamente, associados aos coeficientes específicos de cada função polinomial $\left(\phi_{\mathrm{m}}, \mathrm{m}=0, \mathrm{p}-1\right.$ para $\mathrm{p}$ indicando a ordem do polinômio $)$ e $\mathrm{e}_{\mathrm{ijkl}}$ é o erro aleatório ou medida de erro temporário associado a cada PLC. Os polinômios de Legendre foram padronizados entre -1 e +1 representando o intervalo de 5 a 305 dias de lactação.

Foram realizadas seis análises para a avaliação dos polinômios de Legendre de ordem 3, 4 e 5. Duas formas de ajuste do efeito residual foram avaliadas para cada polinômio. Em uma forma assumiu-se variância residual (VR) constante ou homogênea ao longo da lactação e, na outra forma assumiu-se que a VR era constante dentro, mas heterogênea entre classes de período de lactação, definidas pelos intervalos: 5-49, 50-119, 120-169 e 170-305 dias de lactação.

O modelo acima pode ser escrito na forma matricial por:

$$
\mathbf{y}=\mathbf{X b}+\mathbf{Z a}+\mathbf{Z}_{\mathbf{D}} \gamma+\mathbf{e}
$$

em que y é o vetor das observações; b, o vetor dos efeitos fixos; $\gamma$, o vetor dos coeficientes de regressão aleatória do efeito genético aditivo de animal; $a$, o vetor dos coeficientes de regressão aleatória do efeito permanente de ambiente; e, o vetor de efeito aleatório residual; e X, $\mathrm{Z}, \mathrm{e} \mathrm{Z}_{\mathrm{d}}$ são as matrizes de incidência correspondentes às observações, para efeitos fixos, efeito animal e efeito permanente de ambiente, respectivamente.

Neste modelo assume-se:

$$
\left[\begin{array}{l}
\boldsymbol{\alpha} \\
\boldsymbol{\gamma} \\
\mathbf{e}
\end{array}\right] \sim N(\mathbf{0}, \mathbf{V}), \mathrm{V}=\left[\begin{array}{ccc}
\Lambda_{\mathrm{A}} \otimes \mathrm{A} & 0 & 0 \\
0 & \Lambda_{\mathrm{p}} \otimes \mathrm{I} & 0 \\
0 & 0 & \mathrm{R}
\end{array}\right]
$$

em que $\Lambda_{\mathrm{A}}$ e $\Lambda_{\mathrm{P}}$ são, respectivamente, as matrizes de covariância genética aditiva e de efeito permanente ambiente para os coeficientes de regressão aleatória, A, a matriz dos coeficientes de parentesco entre os animais; I, uma matriz identidade; $\otimes$, o operador de produto direto entre matrizes; e $\mathrm{R}=\operatorname{diag}\left\{\sigma_{e s}^{2}\right\}$, em que $\boldsymbol{s}$ representa as classes de dias em lactação, para ajuste da variância heterogênea entre grupos ao longo da lactação. Assim, $s=1, \ldots, 4$ para as classes: 5-49, $50-119,120-169$ e $170-305$ dias ou ainda $s=1$ quando 
assume-se variância residual homogênea ou constante ao longo da lactação. Os modelos ajustados usando os LP foram identificados por LP seguido da ordem e do número de medidas de erro ajustadas (4 ou 1). Assim LP34 identifica um MRA usando o LP de ordem 3 com ajuste de 4 classes ou medidas de erro.

Os componentes de covariância para os efeitos aleatórios das funções paramétricas e polinômios ortogonais de Legendre foram estimados com um modelo animal usando-se o programa 'DxMRR' (Meyer, 1998c) e o algoritmo de informação média AIREML (Johnson \& Thompson, 1995; Meyer, 1997). Definiu-se como critério de convergência a diferença entre o valor da função de verossimilhança de iterações consecutivas menor que $10^{-4}$. Cada análise foi repetida com os valores estimados na análise anterior até que o valor da função de verossimilhança fosse igual entre duas análises consecutivas.

A avaliação dos diferentes modelos ajustados foi feita por meio da comparação entre os componentes de covariância e parâmetros estimados para as funções ajustadas entre si e relativamente aos valores do logaritmo do máximo da função de verossimilhança $(\log l)$ e dos Critérios de Informação de Akaike $(A I C=-2 \log l+2 p)$, em que $p$ é o número de parâmetros estimados e log l, o logaritmo do máximo da função de verossimilhança (Meyer, 2001).

\section{Resultados e Discussão}

Na Tabela 1 são apresentadas as médias e respectivos desvios-padrão para produção de leite dos controles individuais (PLC) e para a produção de leite acumulada em 305 dias (P305). Na Tabela 2 apresentam-se os componentes de variância e parâmetros genéticos para a PLC e P305. A estimativa do componente de

Tabela 1 - Número de observações, médias e desviopadrão (DP) para a produção de leite nos controles (PLC) e em 305 dias (P305), de primeiras lactações de vacas da raça Gir

Table 1 - Number of records, average and standard deviation (SD) of test day and 305-d milk yield of first lactation Gir cows

\begin{tabular}{|c|c|c|c|c|c|}
\hline \multicolumn{3}{|c|}{$\begin{array}{c}\text { PLC } \\
\text { Test-day milk yield }\end{array}$} & \multicolumn{3}{|c|}{$\begin{array}{c}\mathrm{P} 305 \\
305 d \text { milk yield }\end{array}$} \\
\hline$\overline{\mathrm{PLC}}$ & Média & DP & Lactações & Média & DP \\
\hline$T D$ & Average & $S D$ & Lactations & Average & $S D$ \\
\hline 8.183 & 8,5 & 3,5 & 1.279 & $1.973,6$ & $1.039,1$ \\
\hline
\end{tabular}

variância genética para P305 foi superior ao valor $115.536,40 \mathrm{~kg}^{2}$ obtido por Costa et al. (2004) para a produção de leite na primeira lactação de vacas Gir.

A estimativa da heritabilidade foi igual às obtidas por Verneque et al. (2000b) e por Costa et al. (2004). $O$ resultado de estimativa de heritabilidade para a produção dos $\operatorname{PLC}(0,27)$ superior à obtida para $\mathrm{P} 305$ $(0,22)$ assemelha-se ao relatado por Strabel \& Szwaczkowski (1997), que obtiveram maiores estimativas de heritabilidade para os registros de leite, gordura e proteína dos controles (modelados como caracter único) do que para as produções de leite, gordura e proteína acumuladas em 305 dias de lactação na raça Holandesa. Com esta mesma raça, Olori et al. (1999) também relataram maior estimativa de heritabilidade para as PLC ajustadas com um modelo de repetibilidade do que para a produção de leite acumulada em 305 dias.

No que concerne ao ajuste dos modelos por regressão aleatória, as estimativas de variância residual (VR) obtidas com a FAS foram ligeiramente inferiores às obtidas com a FW, tanto para ME1 como para ME4, provavelmente devido ao ajuste de maior número de parâmetros com a FAS (Tabela 3). Estes resultados coincidem com os relatados por Brotherstone et al. (2000). As variâncias residuais para os grupos 2 e 3 em ME4 foram semelhantes em ambas as funções e sugerem que se possa considerá-las em futuras análises em um só grupo (Olori et al., 1999).

Ainda na Tabela 3 observa-se que as estimativas de VR para os LP decresceram com o aumento da ordem do polinômio. Segundo Meyer (1998a), a redução da VR com o aumento da ordem do modelo está relacionada à sua maior capacidade em modelar as produções do início da lactação. De forma análoga ao observado para as FAS e FW, a VR dos LPs para ME4 foram maiores no início e no final da lactação do que na fase intermediária. As estimativas de VR para o LP54 foram semelhantes àquelas obtidas com a FAS4, mais próximas da estimativa de VR obtida com o MR. As funções de máxima verossimilhança $(\log £)$ aumentaram um pouco com o aumento da ordem do polinômio e com base no Critério de Informação de Akaike (AIC), entre os polinômios ortogonais, o LP54 foi o modelo de melhor ajuste, enquanto a FAS foi melhor que a FW.

As variâncias genéticas entre as FAS e FW (Figura 1) apresentaram tendências semelhantes, de redução de valores entre $4,0-8,0 \mathrm{~kg}^{2}$ ao início da 
Tabela 2 - Estimativa dos componentes de variância genética aditiva (VG), de ambiente permanente (VP), residual (VR), herdabilidades ( ), e repetibilidade ( ) para a produção de leite nos controles (PLC) e em 305 dias, de primeiras lactações de vacas da raça Gir

Table 2 - Estimates of additive genetic (VG), permanent environment (VP), residual (VR), heritability ( ), and repeatability ( ) of test day and 305-d milk yield of first lactation Gir cows

PLC

Test-day milk yield
P305

305 d milk yield

\begin{tabular}{|c|c|c|c|c|c|c|c|}
\hline $\mathrm{VG}$ & $\mathrm{VP}$ & VR & $\hat{h}^{2}$ & & $\mathrm{VG}$ & VR & \\
\hline 1,74 & 3,19 & 1,56 & 0,27 & 0,76 & $121.094,6$ & $439.376,9$ & 0,22 \\
\hline
\end{tabular}

Tabela 3 - Número de parâmetros (NP), estimativa de variância residual (VR), logaritmo da função de verosimilhança ( $€$ ), e Critério de Informação de Akaike (AIC)A para as funções logarítmica e exponencial e polinômios de Legendre de ordem $(\mathrm{P}=3,4$ e 5$)$ para duas classes de erros de medição (ME) ${ }^{B}$

Table 3 - Number of parameters (NP), estimated residual variances (VR) and Log likelihood ( $E$ ) and Akaike information criterion $(A I C)^{A}$ for logarithmic (FAS) and exponential (FW) functions and Legendre polynomials of order $(p=3,4$ and 5$)$ for two measurement error (ME) classes $^{B}$

\begin{tabular}{|c|c|c|c|c|c|c|c|c|c|c|}
\hline \multicolumn{3}{|c|}{$\begin{array}{l}\text { Modelo } \\
\text { Model }\end{array}$} & \multicolumn{3}{|c|}{$\mathrm{FAS}^{1}$} & \multirow[b]{2}{*}{$\mathrm{H}$} & \multicolumn{4}{|c|}{$\mathrm{FW}^{2}$} \\
\hline $\mathrm{ME}$ & $\mathrm{H}$ & $\mathrm{a}$ & $\mathrm{b}$ & $\mathrm{c}$ & $\mathrm{d}$ & & $\mathrm{a}$ & $\mathrm{b}$ & $\mathrm{c}$ & $\mathrm{d}$ \\
\hline$\overline{\mathrm{NP}}$ & 31 & \multicolumn{4}{|c|}{34} & 13 & \multicolumn{4}{|c|}{24} \\
\hline VR & 0,67 & 1,48 & 0,64 & 0,60 & 1.23 & 0,87 & 1,80 & 0,72 & 0,71 & 0,80 \\
\hline$£$ & -6839 & \multicolumn{4}{|c|}{-6792} & -7043 & \multicolumn{4}{|c|}{-6931} \\
\hline AIC & 13739 & \multicolumn{4}{|c|}{13651} & 14112 & \multicolumn{4}{|c|}{13893} \\
\hline
\end{tabular}

\begin{tabular}{|c|c|c|c|c|c|c|c|c|c|c|c|c|c|c|c|}
\hline \multicolumn{2}{|l|}{$\mathrm{LP}$} & \multicolumn{4}{|c|}{3} & \multicolumn{6}{|c|}{4} & \multicolumn{4}{|c|}{5} \\
\hline$\overline{\mathrm{ME}}$ & $\mathrm{H}$ & $\mathrm{a}$ & $\mathrm{b}$ & $\mathrm{c}$ & $\mathrm{d}$ & $\mathrm{H}$ & $\mathrm{a}$ & $b$ & $\mathrm{c}$ & $\mathrm{d}$ & $\mathrm{H}$ & $\mathrm{a}$ & $\mathrm{b}$ & $\mathrm{c}$ & $\mathrm{d}$ \\
\hline$\overline{\mathrm{NP}}$ & 13 & 16 & 21 & 24 & 31 & 34 & & & & & & & & & \\
\hline VR & 0,83 & 1,94 & 0,73 & 0,62 & 1,28 & 0,73 & 1,75 & 0,67 & 0,60 & 1,01 & 0,68 & 1,57 & 0,61 & 0,58 & 1,32 \\
\hline$£$ & -7032 & & & & & -6953 & & -68 & & & -6878 & & & & \\
\hline AIC & 14090 & & & & & 13948 & & 137 & & & 13817 & & 136 & & \\
\hline
\end{tabular}

A AIC = Critério de Informação de Akaike (Meyer, 2001)

${ }^{A} A I C=$ Akaike information criterion, as from Meyer (2001).

$B$ ( $\mathrm{H}$ para $M E=1$ e $a=5-49, b=50-119, c=120-169$ and $d=170-305$ dias para $M E=4)$.

$B$ ( $H$ for $M E=1$ and $a=5-49, b=50-119, c=120-169$ and $d=170-305$ days for $M E=4$ ).

1 Função logarítmica (Logarithimic function): $\mathrm{a}_{0}+\mathrm{a}_{1}(\mathrm{DEL} / 305)+\mathrm{a}_{2}(\mathrm{DEL} / 305)^{2}+\mathrm{a}_{3} \ln (305 / \mathrm{DEL})+\mathrm{a}_{4} \ln (305 / D E L)^{2}$, Ali \& Schaeffer (1987); DEL $=$ days in milk.

2 Função exponencial (Exponential function): $a_{0}+a_{1} D E L+a_{2} \exp \left(a_{3} D E L\right)$, Wilmink (1987).

lactação para valores entre $1,0-1,6 \mathrm{~kg}^{2}$ ao final da lactação. Observou-se porém maiores valores de VG para a FW no início da lactação.

No contexto das VP (Figura 2), observou-se também muita semelhança entre as estimativas obtidas com as FAS e a FW, particularmente após o início (fase intermediária) da lactação. Assim, as diferenças entre as estimativas de VG e VP dependeram da modelagem da VR em ambas as FAS e FW e foram mais aparentes nos extremos do período da lactação. Contrariamente à $\mathrm{FW}$, as estimativas de VG são menores que as de VP para a FAS, o que coincide com as magnitudes relativas destes parâmetros, obtidas com o MR.
Para os LP5 (Figura 3), as VG apresentaram comportamento semelhante, decrescendo do início ao final da lactação, onde apresentam valores próximos a 1,5 $\mathrm{kg}^{2}$. Os valores iniciais para o LP5ME1 foram menores $\left(\sim 5,0 \mathrm{~kg}^{2}\right)$ que os observados para LP5ME4, superiores a $6,0 \mathrm{~kg}^{2}$. As estimativas de VP para estes polinômios são muito semelhantes no período intermediário da lactação (75-275 dias), com estimativas entre $1,7-2,4 \mathrm{~kg}^{2}$. Os maiores valores $\left(4,0 \mathrm{~kg}^{2}\right)$ foram observados ao início e final da lactação para o polinômio LP5ME1. Portanto, a exemplo das FAS e FW, observaram-se diferenças entre os LP para as VG e VP nas extremidades da curva de lactação, para diferentes modelagens da VR. 
As diferenças entre as estimativas de heritabilidade seguiram, como esperado, a mesma tendência dos componentes de variância dos efeitos aleatórios. Com exceção da estimativa $(0,39)$ obtida para a FAS no dia inicial do controle, os valores de heritabilidade (Figura 4) foram semelhantes para as funções polinomiais estudadas e muito elevados, atingindo valores entre $0,49-0,82$ nos primeiros sessenta dias da lactação, decrescendo e atingindo valores próximos de 0,20 no período final da lactação. As

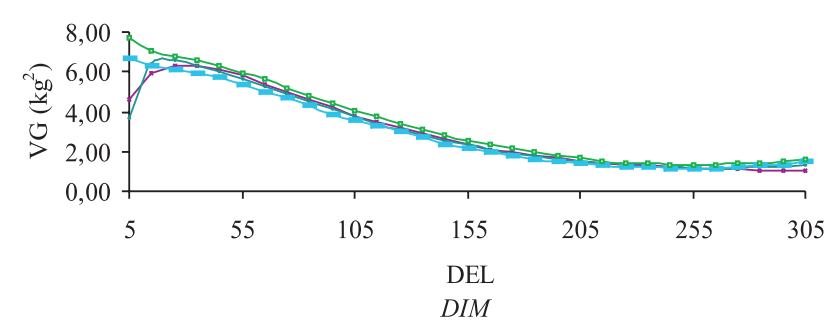

$\multimap$ FASME4 VG $\longrightarrow$ FASME1 VG - FWME4 VG $\multimap$ FWME1 VG

Figura 1 - Estimativas de variâncias genéticas (VG) para a produção de leite nos dias de lactação (DEL) 5 a 305, obtidas com as funções logarítmica (FAS) e exponencial (FW) com duas classes de erro residual (ME1 e ME4).

Figure 1 - Estimates of additive genetic variances (VG) of milk yield for days in milk (DIM) 5 to 305, obtained for logarithmic (FAS) and exponential (FW) functions and two measurement error classes (ME1 and ME4).

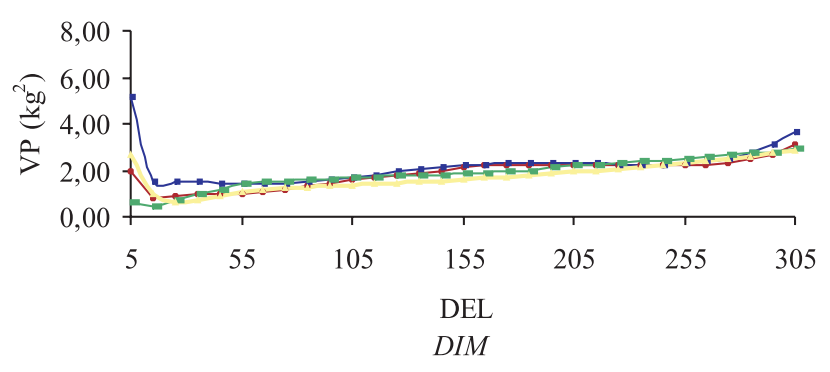

$\longrightarrow$ FASME4 VP — FASME1 VP - FWME4 VP $\longrightarrow$ FWME1 VP

Figura 2 - Estimativas de variâncias de ambiente permanente (VP) para a produção de leite nos dias de lactação (DEL) 5 a 305, obtidas com as funções logarítmica (FAS) e exponencial (FW) com duas classes de erro residual (ME1 e ME4).

Figure 2 - Estimates of permanent environment variances (VP) of milk yield for days in milk (DIM) 5 to 305, obtained for logarithmic (FAS) and exponential (FW) functions and two measurement error classes (ME1 and ME4). estimativas obtidas por Jamrozik \& Schaeffer (1997) e Kettunen et al. (2000) foram também maiores que o esperado quando se usou a função logarítmica para ajuste de modelos de regressão aleatória.

No que concerne aos LP, exceto para o LP51 (Figuras 5 e 6 ) todos os outros modelos resultaram em estimativas de heritabilidade altas e similares entre si, decrescendo de ao redor de 0,70 no início da lactação para 0.30 nos últimos dias de lactação. Este padrão foi contrário àquele reportado por Olori et al. (1999) e Brotherstone et al. (2000).

As estimativas de (co)variância genética e de ambiente permanente e correlações entre os coeficientes de regressão aleatória para FAS e FW com ME4 são apresentadas nas Tabelas 4 e 5 respectivamente. De modo geral foram observadas diferenças significativas nas magnitudes das estimativas de covariância genética e de ambiente permanente e correlações entre os coeficientes para os modelos com ajuste para a VR heterogênea (ME4) ou homogênea (ME1, não mostrados). Este resultado confirma as observações de Olori et al. (1999) de que diferentes modelos de ajuste da VR promovem mudanças nestas estimativas, mas pouco influenciam as estimativas dos componentes de variância genética.

Nenhum padrão específico foi observado para os componentes de (co)variância e de correlação para os coeficientes de regressão estimados para os LP. As estimativas de correlação genética foram em geral elevadas e variaram de $-0,77\left(\mathrm{a}_{0}, \mathrm{a}_{4}\right)$ a $0,84\left(\mathrm{a}_{1}, \mathrm{a}_{4}\right)$ e

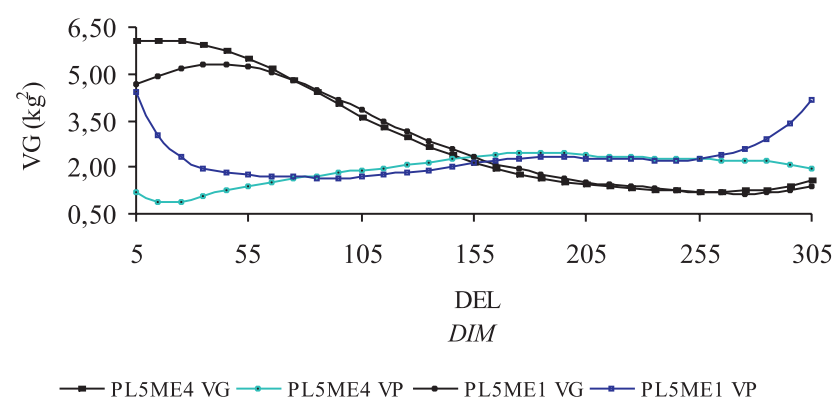

Figura 3 - Estimativas de variâncias genética (VG) e de ambiente permanente (VP) para a produção de leite nos dias de lactação (DEL) 5 a 305, obtidas com polinômios de Legendre de ordem 5 com duas classes de erro residual (ME1 e ME4).

Figure 3 - Estimates of additive genetic (VG) and permanent environment (VP) variances of milk yield for days in milk (DIM) 5 to 305, obtained for Legendre polynomials of order 5 (PL5) and two measurement error classes (ME1 and ME4). 


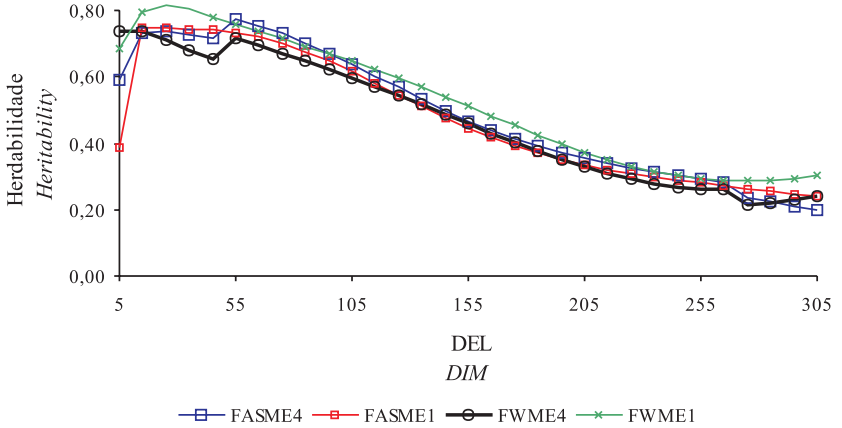

Figura 4 - Estimativas de heritabilidade para a produção de leite nos dias de lactação (DEL) 5 a 305, obtidas as funções logarítmica (FAS) e exponencial (FW) com duas classes de variância residual (ME1 e ME4).

Figure 4 - Estimates of heritability of milk yield for days in milk (DIM) 5 to 305, obtained for logarithmic (FAS) and exponential (FW) functions and two measurement error classes (ME1 and ME 4).

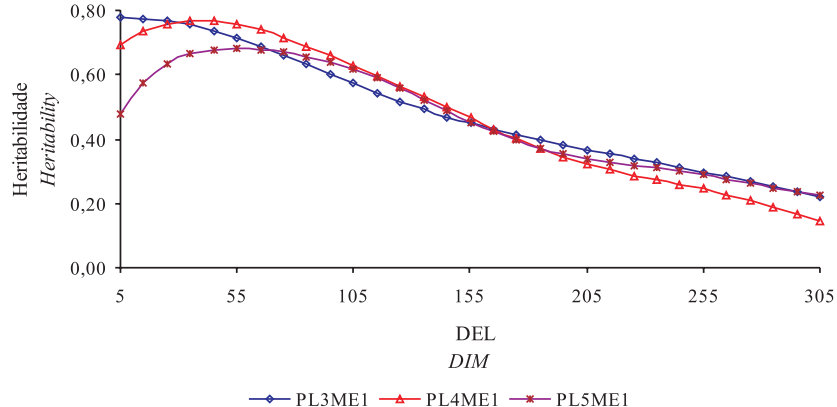

Figura 5 - Estimativas de heritabilidade para a produção de leite nos dias de lactação (DEL) 5 a 305, obtidas com polinômios de Legendre de ordem 3 a 5 com uma medida de erro residual (ME1).

Figure 5 - Estimates of heritability of milk yield for days in milk (DIM) 5 to 305, obtained for Legendre polynomials of order 3 to 5 and ME1.

Tabela 4 - Estimativas ${ }^{A}$ de (co)variâncias genéticas e de ambiente permanente $\left(\mathrm{kg}^{2}\right)$ e correlações entre os coeficientes $\left(a_{i}\right)$ para curva paramétrica de Ali e Schaeffer com ME $=4$

Table 4 - Estimates ${ }^{A}$ of genetic and permanent environment $(c o)$ variance $\left(\mathrm{kg}^{2}\right)$ and correlation between regression coefficients $\left(a_{i}\right)$ for Ali \& Schaeffer logarithmic function and ME=4

\begin{tabular}{|c|c|c|c|c|c|c|c|c|c|}
\hline$a_{0}$ & $a_{1}$ & $a_{2}$ & $a_{3}$ & $a_{4}$ & $a_{0}$ & $a_{1}$ & $a_{2}$ & $a_{3}$ & $a_{4}$ \\
\hline \multicolumn{5}{|c|}{$\begin{array}{l}\text { Genética } \\
\text { Genetic }\end{array}$} & \multicolumn{5}{|c|}{$\begin{array}{l}\text { Ambiente permanente } \\
\text { Permanent environment }\end{array}$} \\
\hline 45,742 & $-0,956$ & 0,829 & $-0,849$ & 0,558 & 18,779 & $-0,899$ & 0,707 & $-0,878$ & 0,403 \\
\hline$-72,080$ & 124,183 & $-0,948$ & 0,770 & $-0,431$ & $-45,742$ & 137,720 & $-0,933$ & 0,704 & $-0,175$ \\
\hline 28,033 & $-52,870$ & 25,020 & $-0,615$ & 0,235 & 25,651 & $-91,665$ & 70,132 & $-0,401$ & $-0,166$ \\
\hline$-14,010$ & 20,931 & $-7,510$ & 5,953 & $-0,900$ & $-7,796$ & 16,923 & $-6,884$ & 4,198 & $-0,791$ \\
\hline 0,910 & $-1,157$ & 0,284 & $-0,529$ & 0,058 & 0,483 & $-0,566$ & $-0,384$ & $-0,447$ & 0,076 \\
\hline
\end{tabular}

A Estimativas de variância na diagonal, estimativas de correlação e covariância acima e abaixo da diagonal, respectivamente.

A Estimates of variance on the diagonals, correlation and covariance estimates above and below the diagonal, respectively.

Tabela 5 - Estimativas ${ }^{1}$ de (co)variâncias genéticas e de ambiente permanente $\left(\mathrm{kg}^{2}\right)$ e correlações entre os coeficientes $\left(a_{i}\right)$ para curva paramétrica de Wilmink com $\mathrm{ME}=4$

Table 5 - Estimates ${ }^{1}$ of genetic and permanent environment (co)variance $\left(\mathrm{kg}^{2}\right)$ and correlation between regression coefficients $\left(a_{i}\right)$ for Wilmink exponential function $(F W)$ and $M E=4$

\begin{tabular}{|c|c|c|c|c|c|c|}
\hline & $\mathrm{a}_{0}$ & $\mathrm{a}_{1}$ & $\mathrm{a}_{2}$ & $\mathrm{a}_{0}$ & $\mathrm{a}_{1}$ & $\mathrm{a}_{2}$ \\
\hline & \multicolumn{3}{|c|}{$\begin{array}{l}\text { Genética } \\
\text { Genetic }\end{array}$} & \multicolumn{3}{|c|}{$\begin{array}{l}\text { Ambiente permanente } \\
\text { Permanent environment }\end{array}$} \\
\hline $\mathrm{a}_{0}$ & 8,485 & $-0,930$ & $-0,433$ & 1,679 & $-0,061$ & $-0,873$ \\
\hline$a_{1}$ & $-0,029$ & 0,001 & 0,403 & $-0,001$ & 0,000 & 0,502 \\
\hline$a_{2}$ & $-2,455$ & 0,008 & 3,784 & $-2,294$ & 0,004 & 4,115 \\
\hline
\end{tabular}

${ }^{1}$ Estimativas de variância na diagonal, estimativas de correlação e covariância acima e abaixo da diagonal respectivamente.

${ }^{1}$ Estimates of variance on the diagonals, correlation and covariance estimates above and below the diagonal respectively.

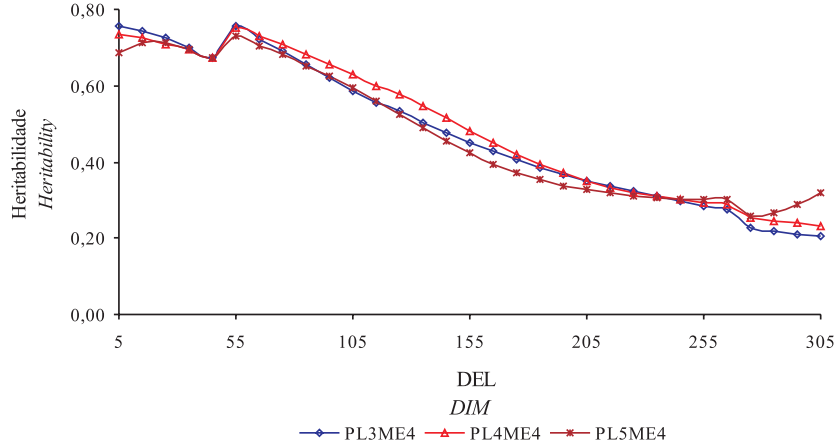

Figura 6 - Estimativas de heritabilidade para a produção de leite nos dias de lactação (DEL) 5 a 305, obtidas com polinômios de Legendre de ordem 3 a 5 com quatro medidas de erro residual (ME4).

Figure 6 - Estimates of heritability of milk yield for days in milk (DIM) 5 to 305, obtained for Legendre polynomials of order 3 to 5 and ME4. 
foram maiores que as correspondentes estimativas para os efeitos permanentes de ambiente para os LP de ordem cinco com ME4 (Tabela 6).

As estimativas de correlação genética entre as PLC em determinados dias de lactação obtidas para as curvas FAS e FW com ME4 são apresentadas na Tabela 7. Observou-se uma redução de valores próximos à unidade entre as PLC adjacentes no início da lactação para valores menores entre as PLC no início e no fim da lactação. Maiores valores foram obtidos com a FW, que também apresentou maior número de estimativas negativas entre as PLC dos extremos da lactação.

De forma semelhante às FAS e FW, o padrão das estimativas de correlação genética foi consistente entre os LP. Na Tabela 8 são apresentadas as estimativas de correlação genética entre as PLC para o LP de ordem cinco. Observa-se redução de valores próximos à unidade entre as PLC adjacentes no início da lactação para valores negativos entre as PLC no início e final da lactação. Estimativas de valor mediano foram observadas na fase intermediária da lactação. Em estudo comparativo entre os polinômios ortogonais e as funções paramétricas (logarítmica e exponencial) na análise de registros das PLC de vacas Holandesas, Brotherstone et al. (2000) observaram que as funções paramétricas produziram um melhor ajuste, mas com estimativas de correlações genéticas negativas entre o período inicial e final da lactação. Correlações genéticas negativas entre os PLC dos extremos do período de lactação também foram relatadas por Jamrozik \& Schaeffer (1997), Rekaya et al. (1999), Kettunen et al. (2000) e López-Romero \& Carabaño

Tabela 6 - Estimativas ${ }^{1}$ de (co)variâncias genéticas e de ambiente permanente $\left(\mathrm{kg}^{2}\right)$ e correlações entre os coeficientes $\left(a_{\mathrm{i}}\right)$ para o polinômio de Legendre de ordem cinco com $\mathrm{ME}=4$

Table 6 - Estimates ${ }^{1}$ of genetic and permanent environment (co)variance $\left(\mathrm{kg}^{2}\right)$ and correlation between regression coefficients $\left(\mathrm{a}_{j}\right)$ for Legendre polynomial of order five and $M E=4$

\begin{tabular}{|c|c|c|c|c|c|c|c|c|c|}
\hline $\mathrm{a}_{0}$ & $\mathrm{a}_{1}$ & $\mathrm{a}_{2}$ & $\mathrm{a}_{3}$ & $\mathrm{a}_{4}$ & $\mathrm{a}_{0}$ & $\mathrm{a}_{1}$ & $\mathrm{a}_{2}$ & $\mathrm{a}_{3}$ & $\mathrm{a}_{4}$ \\
\hline & & $\begin{array}{l}\text { Genétic } \\
\text { Genetic }\end{array}$ & & & \multicolumn{5}{|c|}{$\begin{array}{l}\text { Ambiente permanente } \\
\text { Permanent environment }\end{array}$} \\
\hline 4,228 & $-0,626$ & $-0,222$ & 0,409 & $-0,777$ & 3,382 & 0,544 & $-0,412$ & $-0,088$ & $-0,074$ \\
\hline$-1,589$ & 1,522 & $-0,144$ & $-0,200$ & 0,840 & 0,375 & 0,141 & 0,258 & $-0,375$ & 0,072 \\
\hline$-0,134$ & $-0,052$ & 0,087 & $-0,488$ & 0,014 & $-0,378$ & 0,048 & 0,250 & 0,140 & $-0,449$ \\
\hline 0,161 & $-0,047$ & $-0,028$ & 0,038 & $-0,595$ & $-0,046$ & $-0,040$ & 0,020 & 0,082 & $-0,871$ \\
\hline$-0,161$ & 0,104 & 0,000 & $-0,011$ & 0,010 & $-0,032$ & 0,006 & $-0,053$ & $-0,059$ & 0,056 \\
\hline
\end{tabular}

${ }^{1}$ Estimativas de variância nas diagonais e de correlação e covariância abaixo e acima da diagonal, respectivamente.

${ }^{1}$ Estimates of variance on the diagonals, correlation and covariance estimates above and below the diagonal, respectively.

Tabela 7 - Estimativas de correlações genéticas e heritabilidade das produções de leite de controles em determinados dias de lactação (DEL) obtidas com função logarítmica ${ }^{a}$ (acima da diagonal) e exponencial ${ }^{b}$ (abaixo da diagonal) com quatro medidas de erro residual (ME4)

Table 7 - Estimates of genetic correlation ${ }^{A}$ and heritability of daily milk yields for selected days in milk (DIM) obtained from logarithmic (above the diagonal) and exponential (below the diagonal) for ME4

\begin{tabular}{lcccccccccc}
\hline $\begin{array}{l}\text { DEL } \\
\text { DIM }\end{array}$ & 5 & 25 & 45 & 125 & 165 & 265 & 285 & 305 & $\begin{array}{c}\text { Heritabilidade } \\
\text { Heritability }\end{array}$ \\
\hline 5 & & & & & & & & & & FAS \\
25 & 0,94 & - & 0,87 & 0,79 & 0,64 & 0,56 & 0,15 & 0,06 & $-0,04$ & 0,59 \\
45 & 0,88 & 0,99 & - & 0,93 & 0,83 & 0,39 & 0,30 & 0,22 & 0,74 \\
125 & 0,82 & 0,95 & 0,97 & - & 0,97 & 0,66 & 0,57 & 0,48 & 0,74 & 0,57 \\
165 & 0,76 & 0,88 & 0,92 & 0,98 & - & 0,80 & 0,70 & 0,62 & 0,71 \\
265 & 0,21 & 0,27 & 0,31 & 0,51 & 0,66 & - & 0,97 & 0,95 & 0,44 \\
285 & 0,05 & 0,09 & 0,13 & 0,34 & 0,51 & 0,98 & - & 0,99 & 0,28 \\
305 & $-0,09$ & $-0,07$ & $-0,04$ & 0,17 & 0,35 & 0,94 & 0,98 & - & 0,22 \\
\hline
\end{tabular}

a Função logarítmica (Logarithmic function): $a_{0}+a_{1}(D E L / 305)+a_{2}(D E L / 305)^{2}+a_{3} \ln (305 / D E L)+a_{4} \ln (305 / D E L)^{2}$, Ali e Schaeffer (1987); $\mathrm{DEL}=\mathrm{DIM}=$ days in milk.

${ }^{b}$ Função exponencial (Exponential function): $\mathrm{a}_{0}+\mathrm{a}_{1} \mathrm{DEL}+\mathrm{a}_{2} \exp \left(\mathrm{a}_{3} \mathrm{DEL}\right)$, Wilmink (1987). 
(2003). Em estudo mais abrangente, envolvendo 22 modelos com diferentes critérios de avaliação, LópezRomero \& Carabaño (2003) identificaram o polinômio de Legendre de sexta ordem como o mais indicado, embora modelos com diferentes ordens para a modelagem dos efeitos genéticos e de ambiente também fossem uma escolha adequada para ajuste das PLC de vacas Holandesas na Espanha.

Valores superestimados para heritabilidade foram observados por Kettunen et al. (2000) e Jamrozik \& Schaeffer (1997) e têm sido um outro problema associado ao ajuste de PLC com modelos de regressão aleatória. Meyer (1999) observou que o ajuste dos modelos de regressão aleatória, nas extremidades da curva é problemática, provavelmente devido ao menor número de observações e variação aleatória na partição da variância total. Os primeiros e últimos registros de PLC são periféricos e menos informação estaria disponível para as estimativas de variância próximas a estes dias. Segundo López-Romero \& Carabaño (2003), além da escassez de informações no início e fim da lactação, as estimativas de elevadas variâncias nos extremos da curva e de baixas correlações entre as PLC nesses períodos podem resultar de fatores diferenciados ou adicionais atuando nestes processos, além daqueles comuns à mantença da produção na fase intermediária da lactação.

Uma explicação particular para as elevadas estimativas de heritabilidade obtidas neste estudo poderia ser, portanto, a estrutura dos dados. Não foram impostas restrições às lactações curtas. Pool \& Meuwissen (2000) concluíram que o uso de lactações completas para as estimativas dos componentes de variância por regressão aleatória melhorava a qualidade do ajustamento. Os registros de lactações incompletas podem afetar a ponderação dos pontos de ajuste, porque o modelo tem de extrapolar os registros da lactação.

Outra avaliação comparativa dos modelos estudados pode ser relativamente às estimativas de heritabilidade das PLC nos diversos controles obtidas por análise multicaráter. Todavia, a obtenção de valores pretendidos como reais neste tipo de analise exigiria modelos de relativa complexidade, superparametrizados, com um arquivo de estrutura e tamanho adequados. De toda forma, as estimativas de heritabilidade obtidas por Ledic et al. (2002) poderiam ser assumidas como proxy para as PLC de determinados dias da lactação, embora não se refiram exclusivamente à primeira lactação e resultem da média de análises bicarácter entre pares de PLC. As estimativas de heritabilidade obtidas por Ledic et al. (2002) reduziram de 0,24 a 0,14 para a produção de leite do primeiro ao décimo controle. A estimativa da variância genética reduziu ao longo do período de lactação de aproximadamente 2,0 para $0,95 \mathrm{~kg}^{2}$ (a estimativa obtida neste estudo com o MR foi igual a $1,74 \mathrm{~kg}^{2}$, Tabela 2). A variância residual obtida por Ledic et al. (2002) decresceu de 5 para $3,7 \mathrm{~kg}^{2}$. Neste estudo, a estimativa de VR com o MR foi igual a $1,56 \mathrm{~kg}^{2}$ enquanto as estimativas obtidas por regressão aleatória com ME4 foram entre $1,0-2,0 \mathrm{~kg}^{2}$ e inferiores a $1,0 \mathrm{~kg}^{2} \mathrm{com}$ ME1 (Tabela 3).

Tabela 8 - Estimativas de correlações genéticas ${ }^{1}$ e heritabilidade das produções de leite de controles em determinados dias de lactação (DEL) obtidas com polinômios de Legendre de ordem cinco e duas classes de ME

Table 8 - Estimates of genetic correlation ${ }^{1}$ and heritability of daily milk yields for selected days in milk (DIM) obtained from Legendre polynomial of order five and two classes of ME

\begin{tabular}{|c|c|c|c|c|c|c|c|c|c|c|}
\hline \multirow[t]{2}{*}{$\begin{array}{l}\text { DEL } \\
\text { DIM }\end{array}$} & \multirow[t]{2}{*}{5} & \multirow[t]{2}{*}{25} & \multirow[t]{2}{*}{45} & \multirow[t]{2}{*}{125} & \multirow[t]{2}{*}{165} & \multirow[t]{2}{*}{265} & \multirow[t]{2}{*}{285} & \multirow[t]{2}{*}{305} & \multicolumn{2}{|c|}{$\begin{array}{c}\text { Heritabilidade } \\
\text { Heritability }\end{array}$} \\
\hline & & & & & & & & & $\mathrm{ME}=1$ & $\mathrm{ME}=4$ \\
\hline 5 & - & 0,98 & 0,95 & 0,80 & 0,69 & 0,23 & 0,04 & $-0,19$ & 0,48 & 0,69 \\
\hline 25 & 0,98 & - & 0,99 & 0,88 & 0,78 & 0,31 & 0,11 & $-0,12$ & 0,63 & 0,71 \\
\hline 45 & 0,94 & 0,99 & - & 0,93 & 0,83 & 0,37 & 0,18 & $-0,06$ & 0,68 & 0,67 \\
\hline 125 & 0,76 & 0,87 & 0,93 & - & 0,97 & 0,62 & 0,45 & 0,21 & 0,56 & 0,53 \\
\hline 165 & 0,63 & 0,77 & 0,84 & 0,98 & - & 0,76 & 0,61 & 0,39 & 0,42 & 0,40 \\
\hline 265 & 0,11 & 0,28 & 0,38 & 0,65 & 0,78 & - & 0,97 & 0,88 & 0,28 & 0,30 \\
\hline 285 & $-0,08$ & 0,08 & 0,19 & 0,47 & 0,61 & 0,97 & - & 0,96 & 0,25 & 0,27 \\
\hline 305 & $-0,31$ & $-0,17$ & $-0,07$ & 0,20 & 0,35 & 0,83 & 0,95 & - & 0,22 & 0,32 \\
\hline
\end{tabular}

${ }^{1}$ Estimativas de correlação genética para $M E=4$ e $M E=1$ abaixo e acima da diagonal, respectivamente.

${ }^{1}$ Additive genetic correlation estimates for $M E=4$ and $M E=1$ below and above the diagonal, respectively. 
A despeito do baixo número relativo de dados disponíveis para este estudo, deve-se ainda considerar se o ajuste da PLC na raça Gir por regressão aleatória deveria ser baseado em período de lactação de 305 dias. Sabe-se que as raças zebuínas são caracterizadas por período de lactação menor que o das raças européias (Costa et al., 2000). Assim, é possível que um melhor ajuste seja obtido com um período de lactação menor, ao definir o parâmetro "c" no ajuste da função logarítmica (FAS) e o limite superior da padronização do período de lactação no ajuste dos polinômios de Legendre. Há ainda que se observar a maior variabilidade no início e final da lactação (Tabela 3 ) em relação ao período intermediário na revisão de diferentes períodos para ajuste da VR, em atenção às observações de Olori et al. (1999).

Provavelmente, outro aspecto a considerar é a própria forma da curva de lactação da raça Gir, sem um pico de lactação acentuado e um período curto em si (poucas lactações com duração completa até 305 dias), indicando baixa persistência da produção de leite da lactação. Neste contexto, a viabilidade de se realizar as avaliações genéticas para a persistência da lactação (Jakobsen et al., 2002; Cobuci et al., 2004) pode ser importante para a raça Gir. Atualmente, seis países utilizam os modelos de ajuste da PLC nas avaliações genéticas nacionais (Interbull, 2003) e alguns já implementaram o cálculo de valor genético para persistência da lactação considerada como característica funcional, componente do objetivo de melhoria da eficiência da produção. Portanto, a perspectiva de ajuste da PLC com os modelos de regressão aleatória, em razão de sua potencialidade de maior progresso genético para a produção de leite em particular e eficiência da produção em geral, estimula a continuidade de estudos para viabilizar sua aplicação nos programas de seleção da raça Gir.

\section{Conclusões}

A disponibilidade de maior número de informações por lactação para análise e a estimativa de heritabilidade ligeiramente superior no ajuste da PLC, quando comparada ao ajuste da P305, indicam um potencial para uso destes registros nos procedimentos de avaliação genética de bovinos da raça Gir, considerando-se a perspectiva de maior progresso genético por seleção.
Não houve diferenças marcantes entre as funções paramétricas ajustadas, embora a FAS tenha resultado em menores estimativas de variância residual do que a FW. Entre os polinômios ortogonais de Legendre, os de ordem cinco apresentaram melhor ajuste para os efeitos genéticos e de ambiente permanente. As estimativas de heritabilidade foram menores para o PL5 e a FAS e dependeram da modelagem da variância residual, muito semelhantes para esses modelos.

Portanto, os resultados deste estudo indicam o potencial de uso dos modelos de regressão aleatória para ajuste da PLC, com os modelos LP5 e a FAS apresentando-se como os mais adequados para a modelagem das variâncias genética e de efeito permanente das PLC da raça Gir. Todavia, estudos adicionais são necessários considerando-se algumas particularidades da curva de lactação da raça Gir, objetivando uma avaliação mais precisa de eventuais alternativas de modelos de ajuste da PLC e a viabilização das avaliações genéticas para persistência na lactação nesta raça.

\section{Agradecimento}

À Associação Brasileira de Criadores de Zebu ABCZ e à Coordenação do Programa de Melhoramento do Zebu - PMGZ pela disponibilização dos dados, e à FAPEMIG pelo apoio financeiro ao desenvolvimento desta pesquisa.

\section{Literatura Citada}

ALI, T.E.; SCHAEFFER, L.R. Accounting for covariances among test day milk yields in dairy cows. Canadian Journal of Animal Science, v.67, n.3, p.637-644, 1987.

BOLDMAN, K.G.; KRIESE, L.A.; Van VLECK, L.D. et al. A manual for of MTDFREML: a set of programs to abtain estimates of variances and covariances (DRAFT). Washington: USDA, Agricultural Research Service, 1995. 115p.

BROTHERSTONE, S.; WHITE, I.; MEYER, K. Genetic modeling of dairy milk yield using orthogonal polynomial and parametric curves. Animal Science, v.70, p.407-4015, 2000.

COBUCI, J.A.; EUCLYDES, R.F.; COSTA, C.N. et al. Análises da persistência na lactação de vacas da raça Holandesa, usando produção no dia do controle e modelo de regressão aleatória. Revista Brasileira de Zootecnia, v.33, p.546-554, 2004

COSTA, C.N.; MARTINEZ, M.L.; VERNEQUE, R.S. et al. Heterogeneidade de (co)variância para as produções de leite e de gordura entre vacas puras e mestiças da raça Gir. Revista Brasileira de Zootecnia, v.33, p.555-563, 2004 . 
COSTA, C.N.; MACHADO, C.H.C.; JOSAHKIAN, L.A. et al. Evolução no número de rebanhos e no desempenho produtivo das raças zebuínas em controle leiteiro pela $\mathrm{ABCZ}$ na década de 90. 2000. In: Congresso Brasileiro das Raças Zebuínas, 4, 2000, Uberaba, MG. Anais... Uberaba: Associação Brasileira de Criadores de Zebu, 2000. p.115-120.

HILL, W. G.; BROTHERSTONE, S.; VISSCHER, P. M. Current and future developments in dairy cattle breeding: a research viewpoint. Edinburgh: British Society of Animal Science, 1995.

p. 59-66. (Occasional Publication, 19)

INTERBULL 2003. http://www-interbull.slu.se/eval/framesidaprod.htm. Consultado em Junho/2003.

JAKOBSEN, J.H.; MADSEN, P.; JENSEN, J. et al. Genetic parameters for milk production and persistency for Danish Holsteins estimated in random regression models using REML. Journal of Dairy Science, v.85, p.1607-1616, 2002.

JAMROZIK, J.; SCHAEFFER, L.R. Estimates of genetic parameters for a test day model with random regressions for yield traits of first lactation Holsteins. Journal of Dairy Science, v.80, p.762-770, 1997.

JENSEN, J. Genetic evaluation of dairy cattle using test-day models. Journal of Dairy Science, v.84, p.2803-2812, 2001.

JOHNSON, D.L.; THOMPSON, R. Restricted maximumlikelihood estimation of variance components for univariate animal models using sparse-matrix techniques and average information. Journal of Dairy Science, v.78, p.57-69, 1995.

KETTUNEN, A.; MÄNTYSAARI, E.A.; PÖSÖ, J. Estimation of genetic parameters for daily milk yield of primiparous Ayrshire cows by random regression test-day models. Livestock Production Science, v.66, p.251-261, 2000.

LEDIC, I.L.; TONHATI, H.; VERNEQUE, R.S. et al. Estimativa de parâmetros genéticos, fenotípicos e ambientes para as produçõesde leite no dia de controle e em 305 dias de lactação de vacas da raça Gir. Revista Brasileira de Zootecnia, v. 31, n.5, p.1953-1963, 2002.

LÓPEZ-ROMERO, P.; CARABAÑO, M.J. Comparing alternative random regression models to analyse first lactation daily milk yield data in Holstein Friesian cattle. Livestock Production Science, v.82, p.81-96, 2003

MEYER, K. An 'average information' restricted maximum likelihood algorithm for estimating reduced rank genetic matrices or covariance functions for animal models with equal design matrices. Genetic Selection Evolution, v.29, p.97-116, 1997.

MEYER, K. Estimating covariance functions for longitudinal data using a random regression model. Genetic Selection Evolution, v.30, p.221-240, 1998a.

MEYER, K. Modeling 'repeated' records: covariance functions and random regression models to analysis animal breeding data. In: WORLD CONGRESS OF GENETICS APPLIED LIVESTOCK PRODUCTION, 6., 1998, Armidale. Proceedings... Armidale: University of New England, 1998b. v.25, p.517-520

MEYER, K. "DXMRR" - A program to estimate covariance functions for longitudinal dada by Restricted Maximum Likelihood In: WORLD CONGRESS OF GENETICS APPLIED LIVESTOCK PRODUCTION, 6., 1998, Armidale, Australia, Proceedings... Armidale: University of New England, 1998c. v.27, p.465-466.
MEYER, K. Estimates of genetic and phenotypic covariance functions for postweaning growth and mature weight of beef cow. Journal of Animal Breeding and Genetics, v.116, p.181-205, 1999.

MEYER, K. Estimates of direct and maternal covariance functions for growth of Australian beef calves from birth to weaning. Genetic Selection Evolution, v.33, p.487-514, 2001.

MEYER, K.; HILL, W.G. Estimation of genetic and phenotypic covariance functions for longitudinal or "repeated" records by restricted maximum likelihood. Livestock Production Science, v.47, p.185-200, 1997.

OLORI, V.E.; HILL, W.G.; McGUIRK, B.J. et al. Estimating variance components for test day milk records by restricted maximum likelihood with a random regression animal model. Livestock Production Science, v.61, p.53-63, 1999.

PTAK, E.; SCHAEFFER, L.R. Use of test day yields for genetic evaluation of dairy sires and cows. Livestock Production Science, v.34, p.23-34, 1993

POOL, M.H.; MEUWISSEN, T.H. E. Reduction of the number of parameters needed for a polynomial random regression test-day model. Livestock Production Science, v.64, p.133-14, 2000.

POOL, M.H.; JANSS, L.L.G.; MEUWISSEN, T.H.E. Genetic parameters of Legendre polynomials for first parity lactation curves. Journal of Dairy Science, v.83, p.2640-2649, 2000.

REKAYA, R.; CARABAÑO, M.J.; TORO, M.A. Use de test day yields for the genetic evaluation of production traits in Holstein-Friesian cattle. Livestock Production Science, v.57, p.203-217, 1999.

STRABEL, T.; SZWACZKOWSKI, T. Additive genetic and permanent environmental variance components for test day milk yields in Black-white cattle. Livestock Production Science, v.48, p.91-98, 1997.

SWALVE, H.H. The effect of test day models on the estimation of genetic parameters and breeding values for dairy yield traits. Journal of Dairy Science, v.78, p.929-938, 1995.

SWALVE, H.H. Theoretical basis and computational for different test-day genetic evaluation methods. Journal of Dairy Science, v.83, p.1115-1124, 2000.

VERNEQUE, R.S.; LEDIC, I.L.; MARTINEZ, M.L et al. Programa de melhoramento do Gir Leiteiro. In: SIMPÓSIO NACIONAL DA SOCIEDADE BRASILEIRA DE MELHORAMENTO ANIMAL, 3., 2000, Belo Horizonte. Anais... Belo Horizonte: Universidade Federal de Minas Gerais, 2000a. p.212-218.

VERNEQUE, R.S.; MARTINEZ, M.L.; TEODORO, R.L. Avaliação genética de vacas e touros da raça Gir com base na produção de leite em diferentes estágios da lactação. Revista Brasileira de Zootecnia, v.29, n.4, p.1060-1066, 2000b.

WILMINK, J. B. M. Efficiency of selection for different cumulative milk, fat, and protein yields in first lactation. Livestock Production Science, v.17, p.211-224, 1987. 